\title{
Oscillatory Flow in the Human Airways from the Mouth through Several Bronchial Generations
}

\author{
Andrew J. Banko ${ }^{\mathrm{a}}$, Filippo Coletti ${ }^{\mathrm{b}}$, Christopher J. Elkins ${ }^{\mathrm{a}}$, John K. Eaton ${ }^{\mathrm{a}}$ \\ ${ }^{a}$ Department of Mechanical Engineering, Stanford University, Stanford, CA 94305, USA \\ ${ }^{b}$ Department of Aerospace Engineering and Mechanics, University of Minnesota, Minneapolis, MN 55455, USA
}

\begin{abstract}
The time-varying flow is studied experimentally in an anatomically accurate model of the human airways from the mouth through several generations of bronchial branching. The airway geometry is obtained from the CT scan of a healthy adult male of normal height and build. The three-component, three-dimensional mean velocity field is obtained throughout the entire model using phase-locked Magnetic Resonance Velocimetry. A pulsatile pump drives a sinusoidal waveform (inhalation and exhalation) with frequency and stroke-length such that the mean trachea Reynolds number at peak inspiration is 4200 and the Womersley number is 7. Integral parameters are defined to quantify the degree of velocity profile non-uniformity (related to axial dispersion) and secondary flow strength (lateral dispersion). It is found that the extrathoracic airways significantly modify the tracheal flow and that the flow at the first bifurcation is highly asymmetric. The effect of flow oscillation is to produce time dependent flow features which are asymmetric with respect to the acceleration and deceleration periods surrounding peak inhalation and exhalation. This is most pronounced in regions of separation and on the secondary flow structure, which are sensitive to local attributes of the real anatomy. This is reflected in the integral parameters, which behave non-monotonically between successive bronchial generations. In general, the measured oscillatory flow in a realistic anatomy confirms many trends derived from idealized models but also possesses qualitatively different large scale flow structures as compared to idealized representations of the upper airways.
\end{abstract}

Keywords: respiratory flow, pulsatile flow, experimental fluid mechanics, realistic anatomy, Magnetic Resonance Velocimetry

\section{Introduction}

The flow in the human airways spans a wide range of flow phenomena: for example merging streams, oscillating flow, turbulence, secondary flows, and separation. Understanding the integrated effect of these features in real human anatomies is critical to predicting transport in the respiratory system, which is important for the treatment of respiratory disease, preventing the inhalation of airborne pollutants, and understanding gas exchange.

Various levels of idealization have been assumed in previous studies. Much of the prior work focused on the flow in branching tubes assembled from simple shapes and with leftto-right symmetry in both the bronchial tree and extrathoracic airways (Weibel, 1963; Stapleton et al., 2000). These studies elucidated many fundamental details including secondary flow structure, acceleration through the mouth, orientation and dispersion of the laryngeal jet, and turbulent transport in the larynx (Brouns et al., 2007; Shinneeb and Pollard, 2012; Kleinsteuer and Zhang, 2010). Fresconi and Prasad (2007) studied the flow in a symmetric, planar Weibel bifurcation model using Particle Image Velocimetry (PIV) for both steady and oscillating cases. The Reynolds numbers examined were $O(10)-O(100)$

Email addresses: abanko@stanford.edu (Andrew J. Banko ), fcoletti@umn.edu (Filippo Coletti )

Preprint submitted to International Journal of Heat and Fluid Flow so that the model corresponds to generations 5-13 under normal breathing conditions and 8-16 during high frequency ventilation. During steady inhalation and exhalation, they found that secondary flows were generated due to local bifurcation curvature and that upstream effects did not have a significant influence on the secondary flow structure. The secondary flow strength during inhalation was similar to that during exhalation, and the intensity of secondary flows did not exceed approximately $20 \%$ of the average streamwise velocity even for the highest Reynolds numbers. It was concluded that random angular orientations between generations would determine the importance of augmented dispersion by secondary flows, and that mixing may act equally between inhalation and exhalation. Idealized symmetric models produce symmetric secondary flow patterns and this has been observed in the extrathoracic airways and bronchial tree (Fresconi and Prasad, 2007; Brouns et al., 2007). In order to break the degree of symmetry, Comer et al. (2001) simulated the steady, laminar flow in a Weibel model with planar and $90^{\circ}$ non-planar bifurcations considering a Reynolds number range of 500 to 2000 . In the planar model, all secondary flows displayed reflexional symmetry about the bifurcation plane. Secondary flows were attributed to the Dean mechanism (generation by centrifugal pressure gradients acting on the slow near-wall fluid in curved pipes) and displacement of the flow by the carinal ridge. The strength of secondary flows increased with Reynolds number. At both Reynolds numbers studied a small region of reversed flow developed on the outside

March 19, 2016 
wall of the bifurcation. In each generation, the streamwise momentum distribution (location of maximum velocity) and vortex locations differed due to the influence of the upstream generation. It was also observed that the two daughter generations could have different flow patterns due to the inlet condition imposed by the upstream parent generation. The non-planar bifurcation model produced significantly different secondary flow structure. The symmetry about the bifurcation plane was broken and counter-rotating vortices developed with unequal size; a qualitative feature observed in real anatomies (Banko et al., 2015). Despite achieving a fully three-dimensional flow field with the non-planar branching, these models still do not include realistic features such as variable generation length (approximately the distance between upstream and downstream carinal ridges) and large branching angles (angle the flow turns between a parent and daughter generation). As a result the topology of the flow field may differ from real airway flows, especially in truncated models which neglect the influence of the extrathoracic airways (Choi et al., 2009).

Recently, studies in anatomically accurate models have been stimulated by improvements in manufacturing technology, measurement techniques, and simulation capability. de Rochefort et al. (2007) used magnetic resonance velocimetry to study the steady flow in the proximal thoracic airways with comparison to simulations. Zhang et al. (2012) performed numerical simulations of cigarette smoke inhalation in a subjectspecific lung model. Lambert et al. (2011) conducted a Large Eddy Simulation (LES) in a CT-based airway model, demonstrating left-to-right asymmetry in ventilation and particle deposition patterns. Choi et al. (2009) used LES to study the flow in two subject-specific anatomies at steady inhalation. They demonstrated that removing the extrathoracic airways (mouth to glottis) prevents the formation of the laryngeal jet, changing both the mean streamwise momentum distribution and the level of turbulent kinetic energy. When the constriction at the glottis is entirely removed, the flow in the trachea is essentially laminar. When comparing the two anatomies, the flows differed in detail but shared many general features. In particular, vortical structures are generated in the larynx and the laryngeal jet dominates the dynamics in the trachea.

Time-dependent flow features resulting from the inspiration/expiration cycle are noticeable even for the moderate breathing frequency associated with light activity. The effect of flow oscillation is important when the Womersley number, defined by equation 1 , is greater than order unity:

$$
\alpha=\frac{D_{h}}{2} \sqrt{\frac{\omega}{v}}
$$

$D_{h}$ is the hydraulic diameter of the airway, $\omega$ is the frequency of oscillation, and $v$ is the kinematic viscosity. Examples of both experimental and numerical studies of oscillatory flow are available in the literature, in idealized and real human airways. Jan et al. (1989) studied the oscillatory flow in an idealized bifurcation over a range of oscillatory frequencies and amplitudes. They developed a regime diagram describing the importance of terms in the Navier-Stokes equations and supported the classifications by flow visualization and particle streak velocimetry. Of particular relevance to the present study is the convective regime. In this regime the convective acceleration terms are balanced by either viscous forces or the unsteady inertial term, and secondary flows are generated by transverse pressure gradients due to streamline curvature. Furthermore, if the Reynolds number becomes large enough the flow can be turbulent although transition may not occur at the same critical value as straight pipe flow. Lieber and Zhao (1998) examined the flow in a symmetric Weibel bifurcation model in the laminar, convective regime (generations 3-5 under normal breathing conditions). It was concluded that the oscillatory flow surrounding peak inhalation and exhalation closely resembled that of steady flow at the equivalent flow rate. However, during the acceleration and deceleration periods following or approaching a change in the respiratory phase, the flow structures did not resemble the equivalent steady flow features. The flow structures were also asymmetric about peak inhalation and exhalation, a feature not reproducible by steady flow experiments. Choi et al. (2010) simulated the oscillatory flow in a CT-based human airway model and quantified the effect of convective mixing at flow reversal. They found that for high frequency ventilation, with similar parameters to the present study, the interaction of secondary vortices, counter flow phenomena, and turbulence enhanced mixing in the bronchial tree. Grosse et al. (2007) performed time-resolved PIV at the first bifurcation in a realistic anatomical model of the human airways. The flow was asymmetric in the main bronchi due to the left-to-right asymmetry of the anatomy, and a large vortical region developed on the outside wall of the left main bronchus. The extent of this vortical region was sensitive to the Womersley number and instantaneous Reynolds number, i.e. the acceleration and flow inertia. Soodt et al. (2013) used stereo-scanning PIV to study the oscillating flow at the first bifurcation in a realistic airway model and also found significant differences in flow patterns in the left and right main bronchi. During inhalation the region of maximum velocity in the left main bronchus was skewed towards the inside wall of the bifurcation in a U-shape, while the velocity profile was ellipsoidal in the right main bronchus.

Understanding dispersion mechanisms and developing measures of dispersion based on a known velocity field are of interest for predicting gas/particle transport, designing new mechanical ventilators, or interpreting bolus inhalation tests. Multiple modes of transport are important: advection by the bulk flow, longitudinal dispersion due to gradients in the streamwise velocity, lateral transport by secondary flows, dispersion by turbulent motion, and Brownian diffusion. Turbulent transport is significant in the extrathoracic airways where the Reynolds number is high, and diffusion is relevant for nano-size particles and gases. The flow is less turbulent in the first several generations of the bronchial tree, so longitudinal and lateral transport by the bulk flow may play a more important role. Moreover, the branching airway geometry in this region tends to promote skewed velocity profiles and secondary motion. Finally, the large inter-patient variability of deposition efficiency in studies of aerosol drug delivery suggests that realistic anatomy has important effects on transport in the human airways (Longest 
and Holbrook, 2012).

In the present paper we investigate the three-dimensional phase-averaged velocity field during inhalation and exhalation in an anatomically accurate model of the human airways from the mouth through several generations of bronchial branching. Magnetic Resonance Velocimetry (MRV) is used to obtain the three-component velocity field throughout the entire model volume. The specific objective of this contribution is to quantify the impact of the breathing cycle at realistic respiration frequency on flow structures and dispersion metrics derived from the velocity field in the trachea and bronchial tree. In particular, integral measures are introduced to describe both streamwise and lateral transport. It should be noted that the present study has several limitations. The walls of the airways are rigid, the anatomy terminates at approximately the fifth generation of the bronchi, the imposed breathing waveform is sinusoidal, and the study considers only a single anatomy. The experiments on the oscillatory flow in a realistic airway geometry confirm many trends derived from idealized models, but they also show that the flow includes qualitatively different large scale flow structures as compared to idealized representations of the upper airways. These structures are likely to be important for mixing based on integral measures of the velocity field. While the study of a single human's anatomy is be definition not universal, a healthy adult of normal height and build is likely to possess more representative features than idealized models. It is imperative to understand the dependence of flow structures on the real human anatomy in order to predict the flow and to improve medical devices.

\section{Experimental Methods}

The anatomy was provided by Professor C. Kleinstreuer (North Carolina State University, NC). It was obtained from the CT scan of a 47-year old healthy male, $174 \mathrm{~cm}$ in height and $78 \mathrm{~kg}$ in weight, with no history of respiratory disease. A General Electric 64-slice CT scanner was used to image transverse planes at a resolution of $0.97 \mathrm{~mm} \times 0.97 \mathrm{~mm}$ with an original slice thickness of $2.5 \mathrm{~mm}$. Intermediate slices were created via interpolation yielding a final slice thickness of 1.25 $\mathrm{mm}$. The imaged region was from the extra-cranial skull base to the abdominal region and the data were segmented using the ScanIP software (Simpleware Inc.). The anatomy is shown in Figure 1(a). The most distal bronchi in the model correspond to the eighth generation of branching, while most of the bronchial paths in the model truncate at the fifth generation.

The geometry without any scaling was used to manufacture the solid model depicted in Figure 1(b) by stereolithography at the University of Texas El Paso, TX. It consists of three parts flanged together: an inlet, an extrathoracic piece, and a thoracic piece. The inlet provides flow conditioning to mitigate secondary flows and develop a quasi-plug flow at the entrance to the anatomical section. The extrathoracic piece begins at the middle of the mouth; this truncation was made to avoid the influence of a specific positioning of the lips and teeth. It extends to the mid-trachea where it joins the thoracic piece, which encompasses the rest of the bronchi. The terminal bronchi from each lobe of the lung exhaust into five plena, one for each lobe: right upper lobe (RUL), right middle lobe (RML), right lower lobe (RLL), left upper lobe (LUL), and left lower lobe (LLL). Each plenum is at least 20 times larger in characteristic dimension than the terminal bronchi diameters. Separate plastic tubes connect each plenum to a reservoir. A more detailed description is given in Banko et al. (2015).

Water with the addition of $0.06 \mathrm{~mol} / \mathrm{L}$ of copper sulfate was used as the working fluid in order to maximize signal intensity. The kinematic viscosity at the experimental temperature of $22^{\circ} \mathrm{C}$ is $0.96 \cdot 10^{-6} \mathrm{~m}^{2} / \mathrm{s}$, which is approximately 15 times smaller than that of air. Therefore, dynamic similarity with the flow of air was maintained by adjusting flow velocity and period of oscillation in order to match the desired Reynolds number and Womersley number. The flow rate followed a sinusoidal waveform imposed by a custom volumetric pump, which is an idealization of the real respiratory waveform. Choi et al. (2010) performed LES of the respiratory flow in an anatomically accurate airway geometry using both sinusoidal and triangular inspiratory waveforms. They found that both waveforms gave qualitatively the same results despite having flow rates which differed by $50 \%$, suggesting that the flow features are relatively robust to waveform shape. Additionally, previous studies on the oscillatory flow in idealized and realistic bifurcation models employed sinusoidal waveforms (Choi et al., 2010; Fresconi and Prasad, 2007; Zhang and Kleinstreuer, 2002; Adler and Brucker, 2007; Soodt et al., 2013; Grosse et al., 2007). Therefore, using a sinusoidal profile allows the effects of the anatomy in the present study to be singled out. At peak inspiration/expiration the flow rate was $3.78 \mathrm{~L} / \mathrm{min}$ with a bulk trachea velocity of $U_{T}=0.22 \mathrm{~m} / \mathrm{s}$, which is equivalent to about $60 \mathrm{~L} / \mathrm{min}$ in air. This corresponds to a Reynolds number based on the mean trachea hydraulic diameter of $R e=4200$. The mean diameter is $18 \mathrm{~mm}$ and is calculated by averaging over tracheal cross-sections from approximately two diameters below the glottis to two diameters upstream of the first bifurcation. The oscillation period of the flow was 10 seconds, resulting in a Womersley number of $\alpha=7$. This regime is relevant to breathing during moderate exertion. The imposed flow rate waveform was verified using an ultrasonic flow meter (Transonic Systems Inc. TS410), and the ventilation was found to be nearly symmetric with respect to inhalation and exhalation throughout the bronchial tree. Figure 2 shows the measured waveform in terms of the trachea Reynolds number as a function of the phase in the breathing cycle, along with the ideal sinusoidal waveform. Due to the matched pressure drop in the long return tubes from the plena, each lobe of the lung received $20 \%$ of the total flow rate. The left-to-right division of the flow rate at the first bifurcation is similar to that found in previous studies of lung ventilation, although other proportions have also been reported (Briant and Cohen, 1989; Yin et al., 2013). The right middle lobe is over ventilated and for this reason is excluded from the discussion of the results.

Velocity data were obtained using the method of Magnetic Resonance Velocimetry (MRV) described by Elkins and Alley (2007), using the data acquisition procedure described by Pelc et al. (1994). Detailed justification of the use of MRV to mea- 


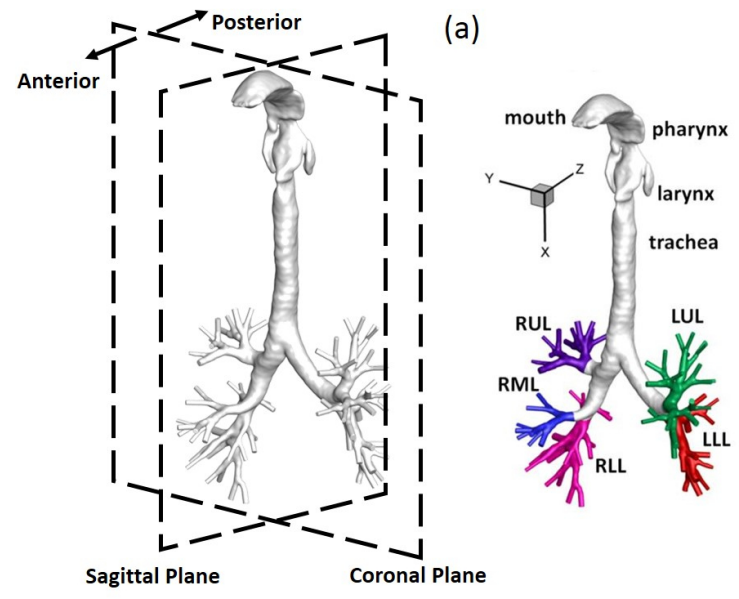

(b)

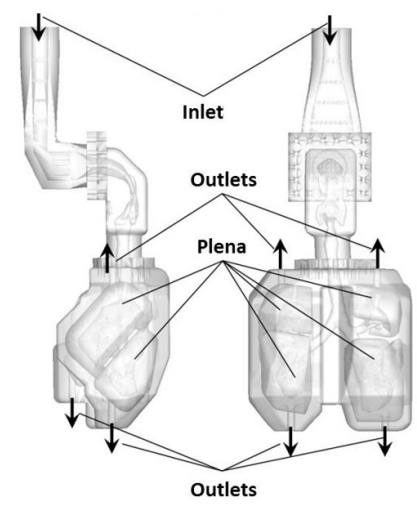

(c)

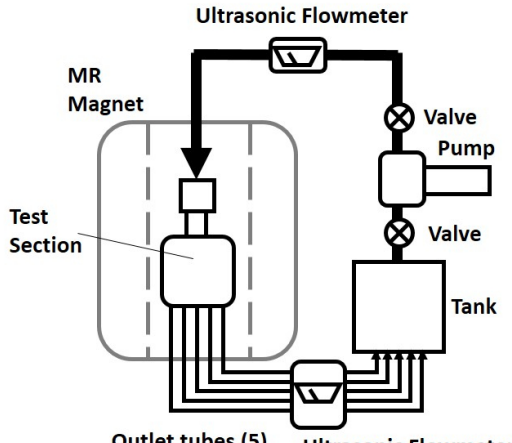

Figure 1: Global isometric view of anatomy with anatomical nomenclature (a), solid model manufactured using stereolithography (b), and flow loop (c).

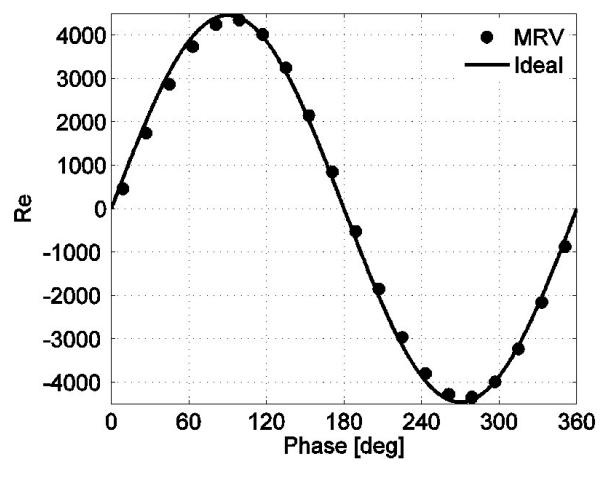

Figure 2: Measured trachea Reynolds number as a function of respiration phase with ideally imposed sinusoidal waveform.

sure this highly three-dimensional flow as compared to optical methods is given in Banko et al. (2015). The MRI pulse sequence used in MRV measures the Fourier transform of the proton spin density (Fourier space is referred to as k-space). By applying the inverse Fourier transform, the real-space proton spin density is recovered. This is a complex function; the amplitude measures the signal strength and the phase encodes the three-dimensional velocity field. For each radio frequency excitation pulse and readout, a line of data in the three-dimensional $\mathrm{k}$-space is collected. This is repeated until all of k-space has been traversed. Since the Fourier transform is non-local, each point in k-space corresponds to the interrogation of the full three-dimensional instantaneous velocity field across $O\left(10^{6}\right)$ real space points. By acquiring the complete set of Fourier coefficients on a fine Cartesian grid of k-space points, collected over a period of many flow through times, a mean velocity field is obtained after applying the inverse transform. In practice, at least two scans are used: one with the flow on and one with the flow off. By subtracting the flow off from the flow on, various sources of error in the measured velocity field, such as eddy currents, can be corrected (Elkins and Alley, 2007).

In order to obtain the phase-locked MRV measurements, a single period of the breathing waveform is subdivided into time increments called segments. The length of each segment sets the intrinsic temporal resolution of the experiment. Within a given segment of the $\mathrm{n}^{\text {th }}$ waveform period only a subset of $\mathrm{k}$ space is acquired, the location in the waveform at which each $\mathrm{k}$-space line was acquired is recorded, and this subset is repeated over all segments in that period. Then a new period begins and the algorithm proceeds to the next region of k-space. Finally, after repeating this procedure over many periods the time-resolved k-space version of the velocity field is obtained. At this point the k-space data are interpolated to a specified number of points in the cycle and the real space velocity field is reconstructed at these points. A more detailed description of the phase-locked MRV technique is given by Markl et al. (2003).

It is difficult to quantify the convergence of the mean velocity field in terms of averages of independent realizations, because the acquisition procedure is fundamentally different from optical and probe based measurements which acquire instantaneous real-space velocity fields (PIV, hot-wire anemometry, etc.). However, extensive validation of MRV has been performed in a wide variety of laminar, turbulent, and pulsatile flows. The review article by Elkins and Alley (2007) summarizes the comparisons made by independent research groups between MRV, LDA, PIV, and CFD simulations. The flows include, but are not limited to, straight and curved pipes, serpentine passages, the human aortic arch, and a backward facing step. More recently phase-locked MRV measurements have been validated against LDA in a bi-stable fluidic oscillator (Wassermann et al., 2013).

The present experiment was performed at the Richard M. Lucas Center for Imaging using a 3 Tesla General Electric whole body scanner with an 8-channel cardiac coil. The flow loop passing through the bore of the magnet is shown in Figure 1(c). Three-component, phase-averaged velocity data were obtained throughout the entire model volume at a spatial resolution of 1 $\mathrm{mm}$ in each Cartesian direction (as compared to the trachea diameter of $18 \mathrm{~mm}$ and the $4 \mathrm{~mm}$ diameter of the smallest reconstructed bronchi). The scanned volume includes both the fluid 
and solid model walls. Wall identification was performed via thresholding based on the signal magnitude: voxels with magnitude greater than 5 times the magnitude of the average noise were identified as fluid. This excludes voxels which contain both the wall and the fluid from the subsequent analysis. The phase-locked measurements were triggered by a digital pulse passing through an ECG converter and into the ECG trigger on the MRI system in order to signal the start of each period of the waveform. Before starting the data acquisition, the pulsatile pump was allowed to run for several waveform periods to eliminate the effects of start-up transients.

Velocity data were obtained in separate scans for the extrathoracic piece and the thoracic piece. The extrathoracic piece was measured using a sagittal slice direction (X-Z plane) with each period of the waveform divided into 12 segments. This gave an intrinsic time resolution of 0.833 seconds. The velocity field at a total of 20 phases were reconstructed using linear interpolation in time, giving a reconstructed time resolution of 0.5 seconds but with added uncertainty due to the interpolation. Data were acquired in two scans with the flow on and the flow off. Each of the 20 reconstructed flow off phases were averaged to reduce noise in the background prior to subtraction, and then the average off case was subtracted from each of the flow on phases. The two background corrected scans were then averaged in order to improve the signal to noise ratio. The total scan time was approximately 45 minutes, which is much longer than all flow time scales.

The thoracic piece was measured with parallel imaging in order to reduce scan time while maintaining the same spatial resolution as the extrathoracic piece. Details of the acquisition procedure can be found in Brau et al. (2008). A coronal slice direction (X-Y plane) was chosen with a reduction factor of 2 in the in-plane phase encode direction. The reconstruction was done using the ARC algorithm, which is described by Beatty et al. (2007). The waveform period was divided into $10 \mathrm{seg}$ ments giving an intrinsic time resolution of 1 second. A total of 20 phases were reconstructed using linear interpolation in time. Three scans were used to produce the final data set: a flow on, a flow off, and a final flow on. Each of the 20 flow off phases were averaged to create a single average off case, and each phase of the first on case was averaged with the corresponding phase of the second on case. Finally, the average off case was subtracted from each of the average on phases, and the two resulting data sets were averaged together. The total scan time was approximately 55 minutes, which is much longer than all flow time scales.

The expected uncertainty in the MRV measurements is given by the formula of Pelc et al. (1994):

$$
\delta_{V}=\frac{\sqrt{2}}{\pi} \frac{V e n c}{S N R}
$$

$V e n c$ is the velocity encoding value which controls the maximum measureable velocity free of aliasing and $S N R$ is the signal to noise ratio. In the extrathoracic scan, the Venc was fixed at $0.6 \mathrm{~m} / \mathrm{s}$ in the coronal plane (X-Y plane), $0.7 \mathrm{~m} / \mathrm{s}$ in the sagittal plane (X-Z plane), and $1 \mathrm{~m} / \mathrm{s}$ in the transverse plane (Y-Z plane). The $S N R$ in this region was 32 . In the thoracic scan,

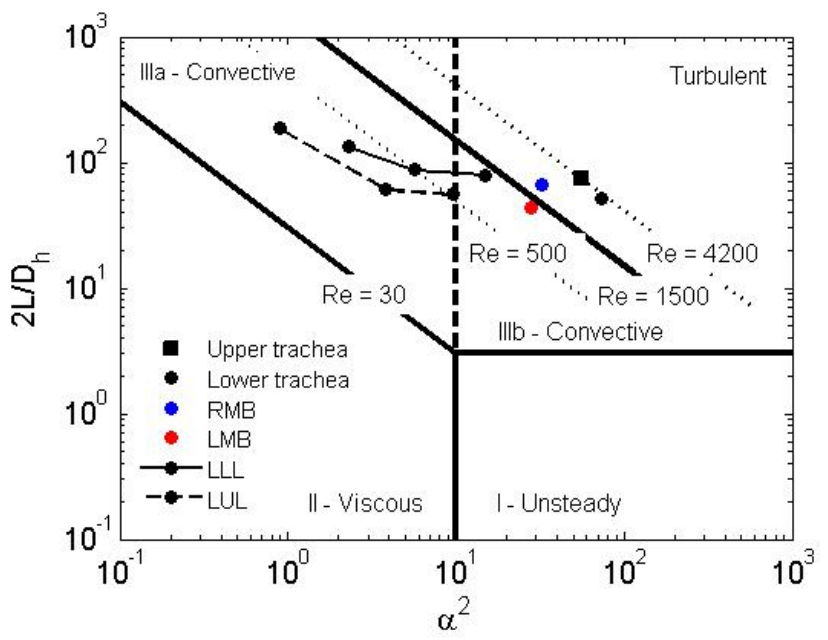

Figure 3: Regime diagram of Jan et al. (1989) classifying the flow the planes shown in Figure 4, Figures 7 - 9, and the bronchial paths through the left lower lobe and left upper lobe.

the Venc was fixed at $0.4 \mathrm{~m} / \mathrm{s}$ in each direction and the $S N R$ was 11 . This results in a maximum uncertainty of about $7 \%$ of the bulk velocity in the trachea at peak inspiration throughout the entire model. The cycle-to-cycle peak flow rate was very repeatable. The variation in the peak flow rate, measured using the ultrasonic flow meter, was approximately $1 \%$. There is also error introduced due to the linear interpolation in time of the k-space data to the 20 selected phases of the breathing cycle. Since the k-space lines within a certain segment of the waveform period are not acquired at exactly the same time in the segment, a direct assessment of the interpolation accuracy based on the reconstructed velocity fields is not possible. However, the error can be estimated by considering the maximum error obtained by approximating a sinusoidal function with linear interpolation. The Fourier coefficients in k-space should be periodic but not necessarily sinusoidal in time, so this is an approximation. For a sinusoidal function $f(t)$ with period $T$, two error metrics are considered:

$$
\begin{aligned}
& e_{1}(\gamma, t, N)=\frac{|(1-\gamma) f(t-\gamma T / N)+\gamma f(t+(1-\gamma) T / N)|}{\max _{t \in\{0, T\}}|f(t)|} \\
& e_{2}(\gamma, t, N)=\left|\frac{(1-\gamma) f(t-\gamma T / N)+\gamma f(t+(1-\gamma) T / N)}{f(t)}\right|
\end{aligned}
$$

$N$ is the number of segments so that $T / N$ is the intrinsic time resolution. It is found that $e_{1}$ maximized over $\gamma \in[0,1]$ and $t \in[0, T]$ is approximately 0.034 for $N=12$ (extrathoracic scan) and 0.049 for $N=10$ (extrathoracic scan). Therefore this interpolation uncertainty is less than that obtained from the signal-to-noise ratio. Likewise, $e_{2}$ is approximately less than or equal to $e_{1}$ for all $\gamma$ and throughout the respiratory cycle except very near flow reversal when it is not well-defined. 


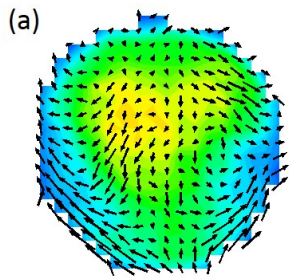

(d)

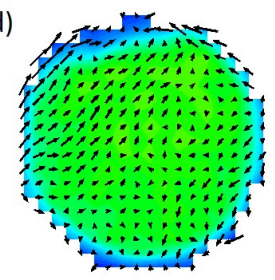

(b)

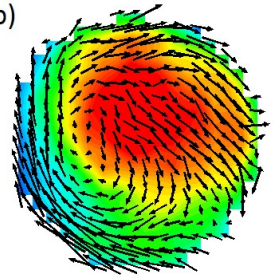

(e)

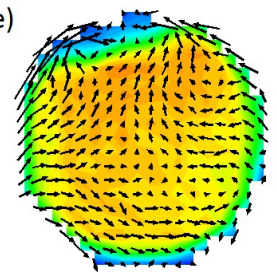

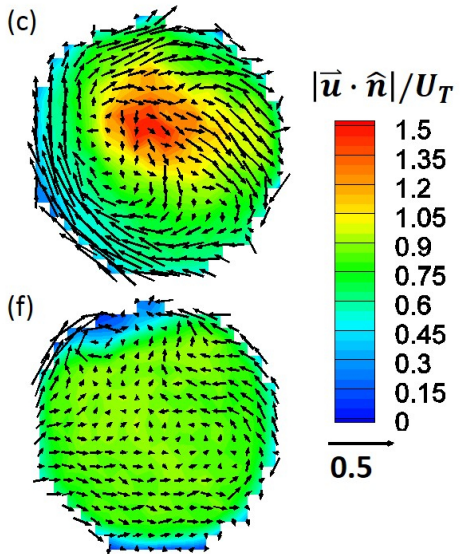

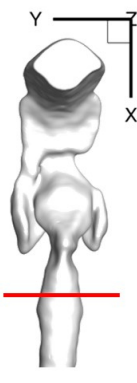

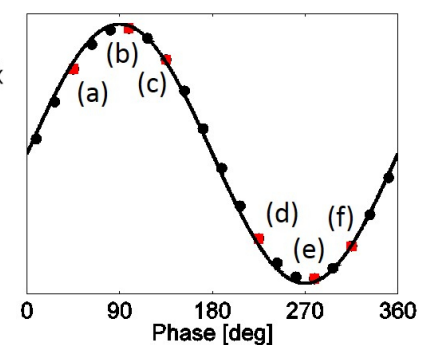

Figure 4: Flow in the trachea showing streamwise velocity contours with in-plane velocity vectors, normalized by the mean speed in the trachea. The view is looking downstream during inhalation with the posterior side of the model at the top of the figure and the anterior side of the model at the bottom of the figure. Selected phases are at $45^{\circ}(\mathrm{a}), 99^{\circ}(\mathrm{b}), 135^{\circ}(\mathrm{c}), 225^{\circ}(\mathrm{d}), 279^{\circ}(\mathrm{e})$, and $315^{\circ}(\mathrm{f})$. These correspond to the phases highlighted by the red squares.

\section{Results and Discussion}

The full data set comprises the phase-averaged, threecomponent velocity at approximately $1.5 \times 10^{6}$ points on a uniform Cartesian grid. This section first discusses qualitative features of the flow in the realistic anatomy, as compared to idealized geometries, with emphasis on the influence of flow oscillation. We then discuss quantitative measures of mixing based on integrals of the phase-averaged velocity field from the first bifurcation through the bronchial tree.

\subsection{Flow Field}

Jan et al. (1989) classify the flow regimes in an airway bifurcation model according to two non-dimensional parameters: the Womersley number, $\alpha$, and the dimensionless stroke length, $2 L / D_{h}$. The stroke length, $L$, describes the axial distance traveled by a fluid particle, and is related to the Reynolds number for a sinusoidal inhalation waveform by $2 L / D_{h}=R e / \alpha^{2}$. For each location in the airways analyzed below, Figure 3 displays the corresponding regime on the diagram proposed by Jan et al. (1989). The trachea, left main bronchus, and right main bronchus are nominally in the convective zone IIIb. In this regime the convective acceleration terms and the unsteady acceleration term are of leading importance in the Navier-Stokes equations. Additionally, the Reynolds number in these regions is high enough so that the flow is expected to be turbulent. Moving further into the bronchial tree, the local Womersley number decreases while the dimensionless stroke length remains the same order of magnitude. Therefore, the flow transitions from zone IIIb to convective zone IIIa between bronchial generations two and three. In zone IIIa, the convective acceleration terms remain important, but the viscous terms become next in importance. This regime has been classified as quasisteady by previous studies: the velocity field during the breathing cycle is approximately represented by the equivalent steady flow (Jan et al., 1989; Zhang and Kleinstreuer, 2002; Lieber and Zhao, 1998). Throughout the present anatomy, the streamwise and secondary velocity components of the oscillatory flow at peak inhalation are found to closely match those in the steady flow experiment of Banko et al. (2015), which used the same anatomy and trachea Reynolds number. This finding is in agreement with previous studies on idealized and real anatomies over the range of Womersley and stroke length parameters in this flow (Zhang and Kleinstreuer, 2002; Grosse et al., 2007; Kleinsteuer and Zhang, 2010). Therefore, the following results focus on the oscillatory nature of the flow.

Figure 4 shows contours of streamwise velocity with secondary flow vectors at a plane located one trachea diameter downstream of the glottis for phases surrounding peak inspiration and peak expiration. In this figure and subsequent ones showing cross-sectional planes, the orientation of the crosssections are such that the reader is looking downstream during inhalation with the posterior side of the model at the top of the figure and the anterior side at the bottom of the figure. Also, the selected inspiratory/expiratory phases are the same for each figure and correspond to the phases highlighted on the breathing waveform by the red squares. The streamwise component shows some similarity between the acceleration and deceleration phases surrounding peak inspiratory flow. Although the flow is slower around the periphery of the cross-section during the accelerating inspiratory phase, the center of the laryngeal jet remains roughly in the same location across panels (a)-(c) of Figure 4. However, the secondary flows differ greatly during the acceleration and deceleration periods. During peak inspiration a strong single-sided streamwise swirl develops in the trachea as a result of the complex recirculation upstream in the larynx and persists down to the first bifurcation, a feature not observed in studies on idealized symmetric geometries. Brouns et al. (2007) examined the influence of the glottic aperture on the tracheal flow using a symmetric extrathoracic airway model and observed streamlines with reflexional symmetry about the sagittal plane in the trachea. Choi et al. (2009) used large eddy simulation to examine the effect of upper airway truncation on the flow in the trachea and found that the airways above the glottis were crucial for generating turbulence in the trachea. The swirl is not yet formed in Figure 4(a) during acceleration, but 


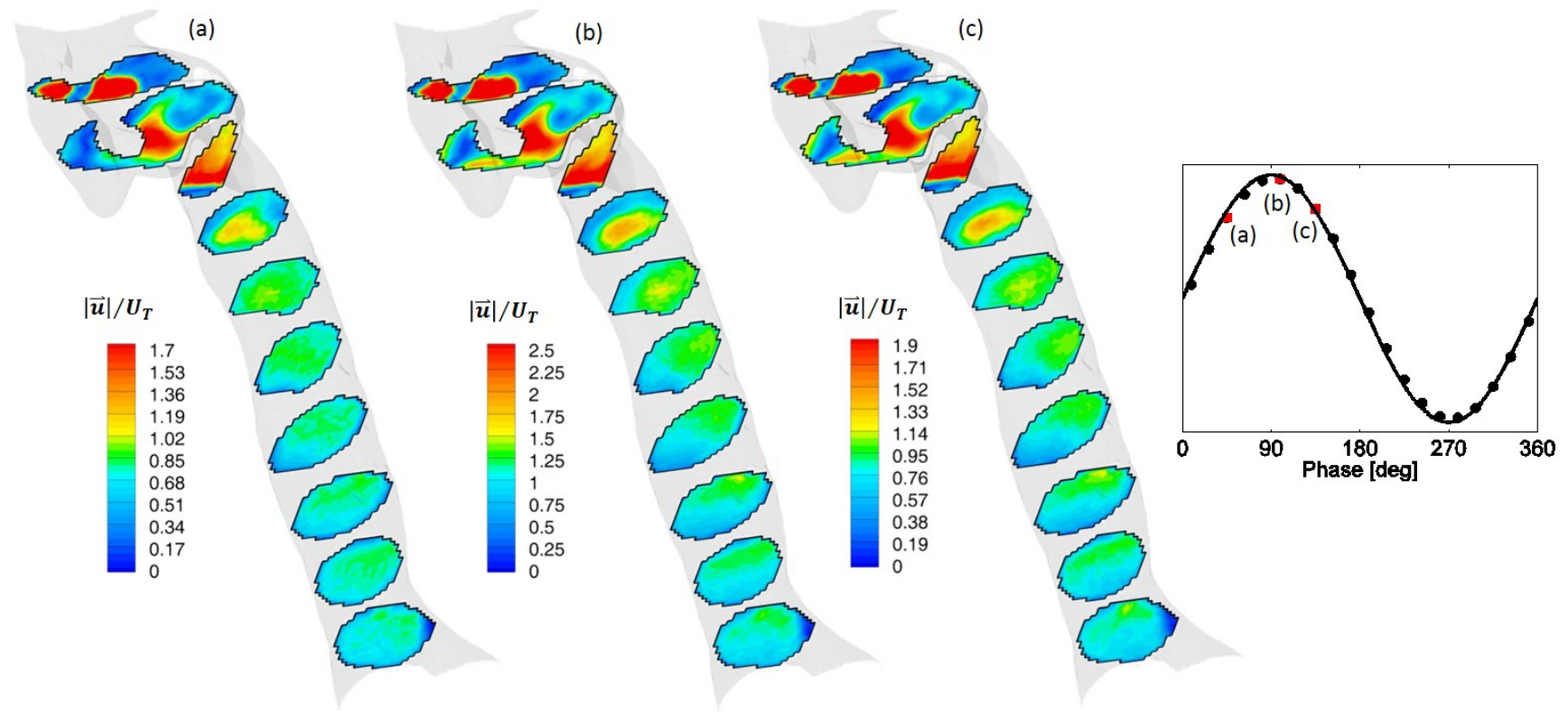

Figure 5: Contours of velocity magnitude during inspiration normalized by the mean speed in the trachea. Planes begin in the supraglottic space and progress downstream to the first bifurcation. Note the change in color scales between the panels. The light gray surface is the boundary of the anatomy. Selected phases are at $45^{\circ}$ (a), $99^{\circ}$ (b), and $135^{\circ}$ (c). These correspond to the first three phases highlighted by the red squares.

lingers during deceleration as seen in Figure 4(c). As a result, the secondary flows are asymmetric in time. Previous results for bronchial bifurcations in the regime IIIb (Jan et al. (1989), idealized bifurcation; Grosse et al. (2007), realistic first bifurcation) similarly display a lack of organized secondary flow structure during acceleration and the sudden onset of secondary flows near peak inhalation which then remain during the deceleration period. Panels (d)-(f) of Figure 4 show the flow during expiration. Due to the distance of this cross-section from the first bifurcation, the secondary flow components during exhalation have decayed appreciably and the streamwise momentum distribution is fairly uniform, similar to that expected for developed turbulent pipe flow.

The effect of the inspiration cycle on the momentum distribution along the trachea is shown in Figure 5. Streamwise normal planes with contours of velocity magnitude are plotted starting from above the glottis and continuing downstream to the first bifurcation. The color scale during each phase of inspiration is modified so that the same qualitative flow structures are highlighted in Figure 5(a)-(c). During flow acceleration, peak inspiration, and flow deceleration a jet-like structure forms in the supraglottic space due to an area constriction at the epiglottis (Banko et al., 2015). The flow continues to accelerate through the triangular shaped glottis and becomes the laryngeal jet in the trachea. As mentioned previously, the location of the jet just downstream of the glottis remains relatively stationary throughout inspiration, and the jet mostly dissipates within several tracheal diameters. During the peak inspiration and deceleration phases, however, the region of high momentum further downstream (green contours in Figure 5) impacts the side wall of the trachea. The stronger streamwise swirl during these phases then advects the high momentum region around to the anterior side of the trachea by the time the flow reaches the first bifurcation. This is in contrast to the acceleration phase during which this region is centered left-to-right and biased towards the anterior side of the trachea along most of its length. The momentum distribution in the trachea is clearly influenced by the upstream airways as observed by Choi et al. (2009) in another realistic anatomy, and sets the inlet condition to the first bifurcation throughout the breathing cycle.

Contours of normalized vorticity magnitude are shown in Figure 6 for a plane through the first bifurcation. There is spatial asymmetry when comparing the flow in the left and right main bronchi (based on physiological convention, the left main bronchus is on the right of the figure and the right main bronchus is on the left of the figure). Due to a tilt in the trachea, the flow during inhalation is better aligned with the right main bronchus and no vorticity is shed from the walls. Conversely, the flow separates from the upper end of the left main bronchus where the streamline curvature is large and adverse pressure gradients due to the diverging bifurcation wall act on the boundary layer fluid. Significant vorticity is shed into this generation. Comer et al. (2001) simulated the flow in an idealized Weibel bifurcation model. At the first bifurcation, the flow divided symmetrically left-to-right. A small region of reversed flow developed along both outside walls of the bifurcation, resulting is some blockage of the streamwise flow, but secondary flows in the daughter generations were not attributed to this event. Grosse et al. (2007) observed a spatially asymmetric distribution of vorticity at the first bifurcation in their patientspecific anatomy, and the vortical region emanating from the outside left wall of the bifurcation extended well into the left main bronchus. During exhalation the vorticity patterns are also markedly different in the two main bronchi. The short length of 

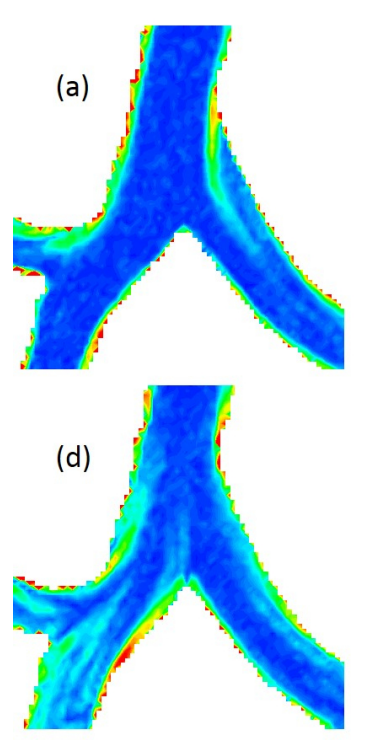
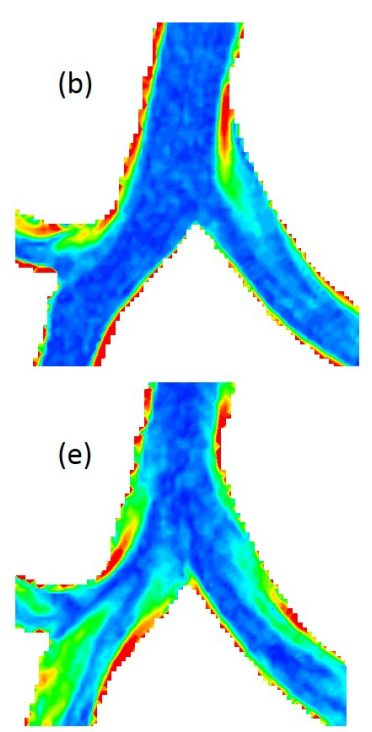
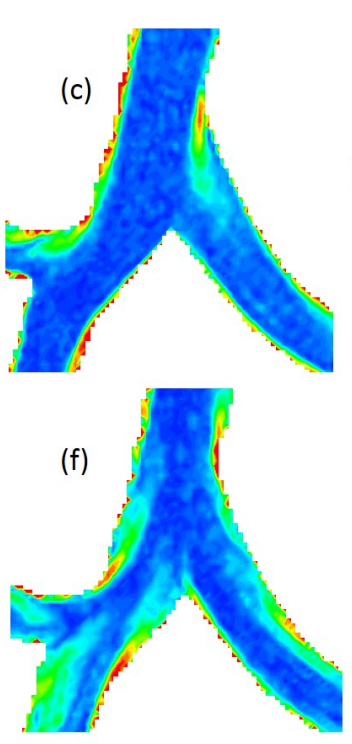

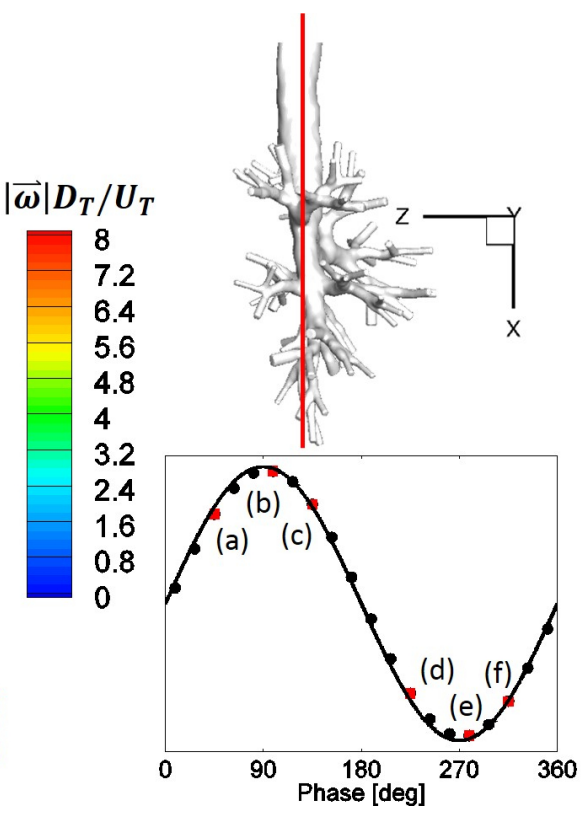

Figure 6: Contours of vorticity magnitude normalized by the mean trachea diameter and speed. The anatomy is viewed from the anterior side, so the right main bronchus is on the left side of the figure and the left main bronchus is on the right side of the figure. Selected phases are at $45^{\circ}$ (a), $99^{\circ}$ (b), $135^{\circ}$ (c), $225^{\circ}$ (d), $279^{\circ}$ (e), and $315^{\circ}$ (f). These correspond to the phases highlighted by the red squares.

the right main bronchus and the high branching angle of the flow entering from the right upper lobe produces strong secondary flows in this region. In contrast, examination of the vector fields suggests that the secondary flow in the left main bronchus is mostly due to the Dean mechanism (see discussion below).

The flow at the first bifurcation also exhibits time asymmetry, similar to that observed upstream in the trachea. This is consistent with the fact that the flow at the first bifurcation is in the convective IIIb regime. Secondary flows develop due to centrifugal acceleration of the streamwise velocity and the flow is not quasi-steady due to the importance of the unsteady acceleration term (Jan et al., 1989; Lieber and Zhao, 1998). During the acceleration leading to peak inhalation, the high vorticity region along the left wall of the bifurcation is elongated and confined along the edge of the separated region. During deceleration the high vorticity region in the same location becomes shorter and diffuse. Therefore lateral dispersion by the secondary flows at the first bifurcation would likely vary across inhalation at the present Womersley number. Similarly, the vorticity distribution shed from the walls during exhalation becomes diffuse within the deceleration period. Grosse et al. (2007) also cited time asymmetry of their elongated vortical region.

Figures 7-9 give a more detailed view of the flow field at planes above the first bifurcation and in the left and right main bronchi. Each figure plots contours of the local streamwise velocity magnitude with secondary flow velocity vectors overlaid for several phases during inhalation and exhalation. Figure 7 shows the flow above the first bifurcation where the cross sectional area is expanding relative to the area of the trachea. During the acceleration phase of inspiration (Figure 7(a)), the fast fluid in the streamwise direction is biased towards the anterior and left side of the trachea. The stronger secondary flows during peak inspiration and the deceleration period then advect this region towards the right side of the trachea (Figure 7(c)). Note the crescent-like shape of the high velocity contours. This skewed streamwise momentum distribution would be responsible for the streamwise dispersion by the phase-averaged flow field during inhalation. The plane in Figure 7 also intersects the separation bubble which forms on the left side of the trachea. During exhalation the merging streams from the two main bronchi result in a more uniform distribution of momentum across this plane. This is reminiscent of the relatively uniform $\mathrm{m}$-shaped profile which develops after the merging of two bronchial flows during exhalation in idealized anatomies Zhang and Kleinstreuer (2002). Once peak exhalation occurs, the secondary flow structure is composed of a single strong vortex and surrounding streaming flow. There is no evidence of the two counter-rotating vortex pairs which develop in symmetric bifurcation models (Jan et al., 1989; Fresconi and Prasad, 2007; Zhang and Kleinstreuer, 2002). The dominant vortex in the trachea appears to be due to an imbalance in the vorticity advected from the left and right main bronchi, as discussed below. Similar to the inspiration phases, the secondary flows are not yet formed during the acceleration phase, are most intense at peak exhalation, and linger during the deceleration phase.

The flows at the start of the left main and right main bronchi are shown in Figures 8 and 9, respectively. Many of the typical characteristics of the upstream oscillatory flow are again evident in these regions. The distribution of momentum is skewed towards the inside wall of the left main bronchus in agreement with studies on idealized geometries (Kleinsteuer and Zhang, 2010). During inhalation this is due to the deflection of high momentum tracheal fluid by the carinal ridge and also due to 

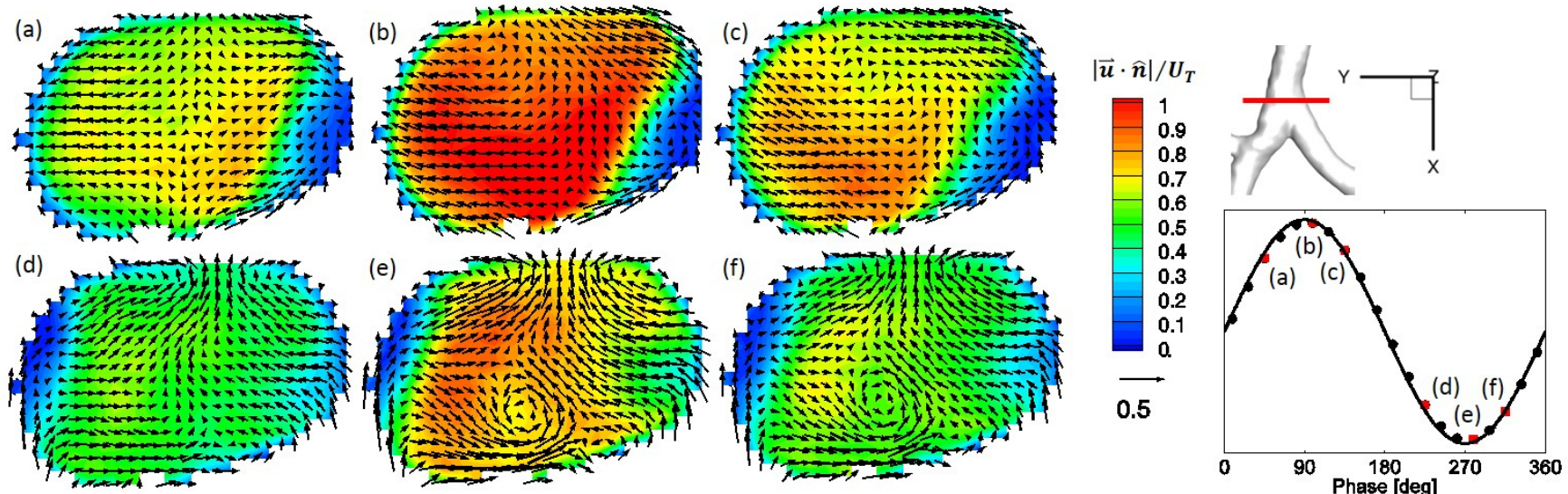

Figure 7: Flow above the first bifurcation showing streamwise velocity contours with in-plane velocity vectors, normalized by the mean speed in the trachea. The view is looking downstream during inhalation with the posterior side of the model at the top of the figure and the anterior side of the model at the bottom of the figure. Selected phases are at $45^{\circ}$ (a), $99^{\circ}$ (b), $135^{\circ}$ (c), $225^{\circ}$ (d), $279^{\circ}$ (e), and $315^{\circ}$ (f). These correspond to the phases highlighted by the red squares.

(a)

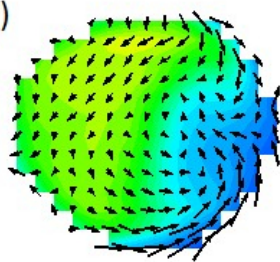

(d)

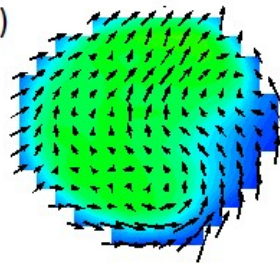

(b)

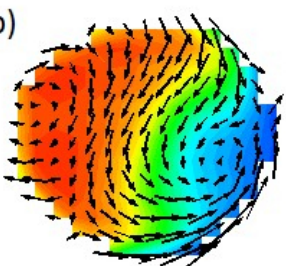

(e)

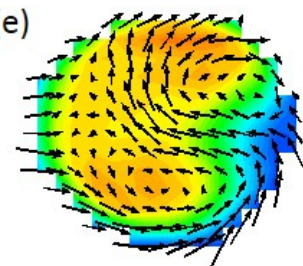

(c)

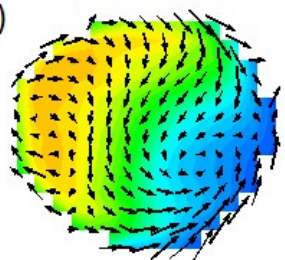

(f)

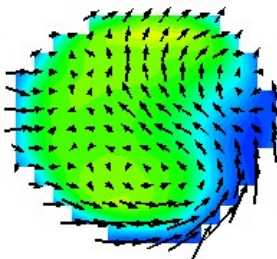

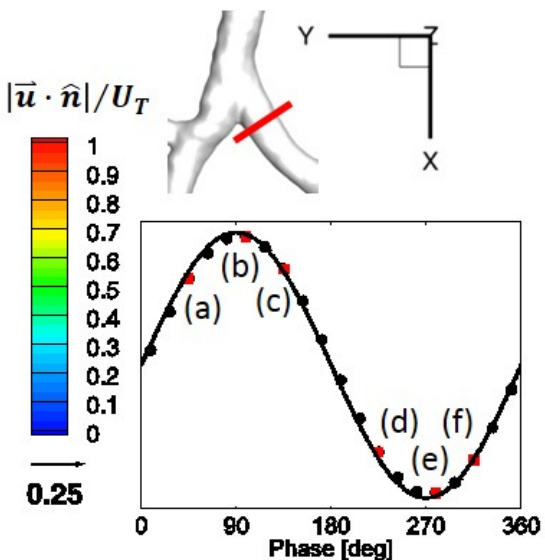

Figure 8: Flow in the left main bronchus showing streamwise velocity contours with in-plane velocity vectors, normalized by the mean speed in the trachea. The view is looking downstream during inhalation with the posterior side of the model at the top of the figure and the anterior side of the model at the bottom of the figure. Selected phases are at $45^{\circ}$ (a), $99^{\circ}$ (b), $135^{\circ}$ (c), $225^{\circ}$ (d), $279^{\circ}$ (e), and $315^{\circ}$ (f). These correspond to the phases highlighted by the red squares.

(a)

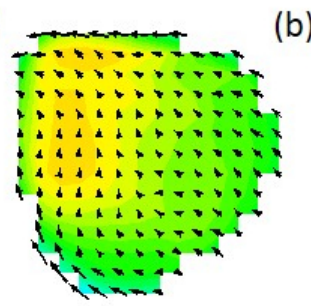

(a)

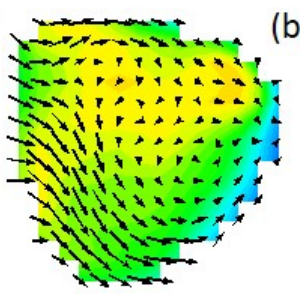

(b)

(b)

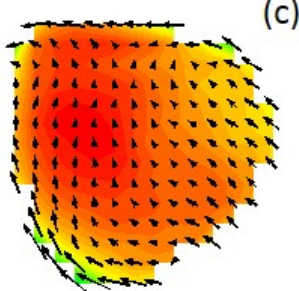

(c)
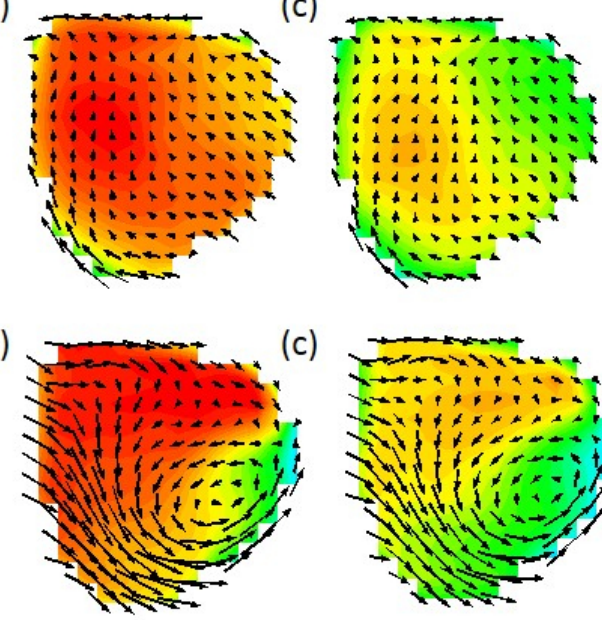

(c)

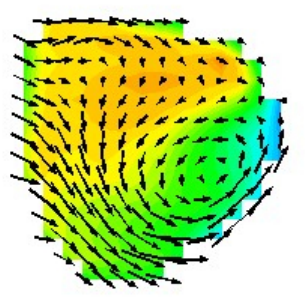

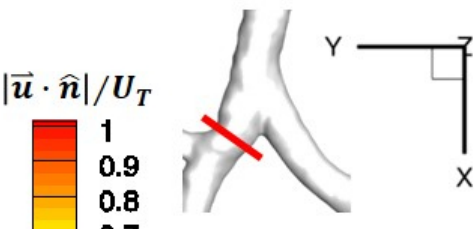

0.7

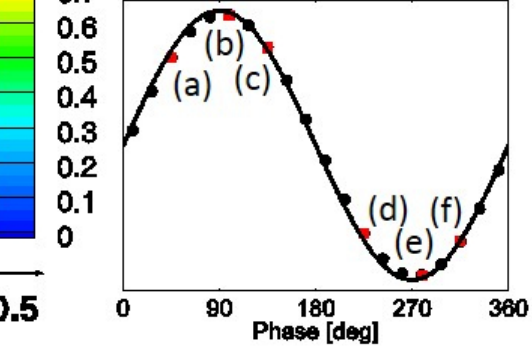

Figure 9: Flow in the right main bronchus showing streamwise velocity contours with in-plane velocity vectors, normalized by the mean speed in the trachea. The view is looking downstream during inhalation with the posterior side of the model at the top of the figure and the anterior side of the model at the bottom of the figure. Selected phases are at $45^{\circ}(\mathrm{a}), 99^{\circ}$ (b), $135^{\circ}$ (c), $225^{\circ}$ (d), $279^{\circ}$ (e), and $315^{\circ}$ (f). These correspond to the phases highlighted by the red squares. 
blockage by the flow separation, similar to the observations of Comer et al. (2001). During exhalation, the streamwise flow displays two peaks on the anterior and posterior sides of the airway. This pattern develops due to the secondary flows arising from the Dean mechanism in agreement with idealized models (Lieber and Zhao, 1998; Comer et al., 2001). In the right main bronchus, the streamwise momentum is more centered and uniform because the tilted trachea is more aligned with this generation. This was also observed in the subject-specific anatomy of Soodt et al. (2013) which showed an ellipsoidal velocity profile in the right main bronchus and a U-shaped velocity profile in the left main bronchus, but not at the first bifurcation of symmetrically bifurcating models (Comer et al., 2001).

The influence of the real anatomical features on the secondary flow structure is particularly evident. First, there is a streamwise vortex pair during both inhalation and exhalation in the left main bronchus. During exhalation (Figure 8(d)-(f)) the positions of the vortices are similar to those generated by the Dean mechanism. Additional counter-rotating vortices have been observed for oscillating flow (Lieber and Zhao, 1998) and are attributed to the mechanism of Lyne (1971) - see also the recent work on oscillating flow in curved pipes by Bulusu et al. (2007). Lyne-type vortices are predicted to occur at Womersley numbers greater than 12.9, but Lieber and Zhao (1998) cite their influence in a model lung bifurcation at a Womersley number of 4.9. Such vortices are not observed in the current experiment. Although this may be due to the experimental resolution, it is clear that the dominant vortices during exhalation resemble Dean vortices, which is the commonly cited mechanism for secondary flows in the bronchial tree (Kleinsteuer and Zhang, 2010). During inhalation the vortex cores in the left main bronchus are instead rotated approximately $90^{\circ}$ in the plane relative to Dean vortices. This is not observed in idealized airway models of the first bifurcation and arises because, during inhalation, the secondary flows in this region are caused by the fact that the three-dimensional separated region upstream sheds vorticity into the bronchus. The strengths of these vortices are higher during the deceleration period of inhalation than during the acceleration period. Therefore, while the Dean vortex formation is consistent with the curved flow paths in some locations of the bronchial tree, other mechanisms may be responsible for the secondary flows depending on the local geometry. In the right main bronchus (Figure 9) the secondary flows are relatively weak during inhalation. There is no upstream separation on the right side of the bifurcation and the flow is not significantly deflected by the carinal ridge, so there are no strong accelerations due to flow turning (and therefore transverse pressure gradients) to produce secondary flows. During exhalation a strong vortex forms due to the merging flow from the right upper lobe at a high branching angle into this generation. This is in contrast to the symmetrically merging bronchi studied by Fresconi and Prasad (2007) which produced counter-rotating vortex pairs. This single-sided vortex is responsible for the dominant vortex in the trachea during exhalation.

\subsection{Integral Parameters}

In order to quantify the dispersion caused by the phaseaveraged velocity field, we introduce integral parameters that characterize the in-plane variability of the streamwise and transverse velocities at a given cross-section of the airways. These parameters are defined as:

$$
\begin{aligned}
& I_{1}=\left(\frac{\iint_{A}(\mathbf{u} \cdot \hat{\mathbf{n}})^{2} d A}{U_{T}^{2} A}\right)^{1 / 2} \\
& I_{2}=\left(\frac{\iint_{A}\|\mathbf{u}-(\mathbf{u} \cdot \hat{\mathbf{n}}) \hat{\mathbf{n}}\|^{2} d A}{U_{T}^{2} A}\right)^{1 / 2} \\
& D=\frac{\iint_{A}(\mathbf{u} \cdot \hat{\mathbf{n}})^{2} d A}{Q^{2} / A}-1 \\
& E=I_{2} / I_{1}
\end{aligned}
$$

Here $\mathbf{u}$ is the phase-averaged velocity vector, $\hat{\mathbf{n}}$ is a unit vector normal to the cross-section, $A$ is the cross-sectional area, $U_{T}$ is the mean speed in the trachea at peak inspiration, and $Q$ is the instantaneous flow rate through the cross-section of interest. $I_{1}$ and $D$ describe the streamwise flow uniformity (which correlates with axial dispersion), where $I_{1}$ is related to the streamwise momentum flux and $D$ is the momentum distortion parameter (Padilla, 2012). It is important to distinguish between the information contained in $I_{1}$ and $D$. $I_{1}$ is the square root of the total momentum flux through a cross-section, and is normalized by a single (global) velocity scale. Therefore, it measures the absolute amount of streamwise transport. In regions with high values of $I_{1}$, fluid elements are being transported more rapidly downstream. $D$ measures the excess streamwise momentum flux relative to a plug flow carrying the same instantaneous flow rate. It is dependent primarily on the shape of the streamwise velocity profile and not on the absolute flow rate. For instance, $D$ is $1 / 3$ in laminar pipe flow and about 0.02 in turbulent pipe flow, regardless of the actual flow rate. Regions with high values of $D$ would tend to cause dispersion in the streamwise direction due to streamwise velocity gradients.

$I_{2}$ and $E$ describe the strength of secondary flows, which would affect lateral dispersion. The parameter $I_{2}$ is a measure of the root mean square velocity of the secondary flows in the cross-section of interest, normalized by a single (global) velocity scale, and therefore measures the absolute strength of secondary flows. Regions with the highest values of $I_{2}$ correspondingly have the highest secondary flow velocity components. The parameter $E$ indicates the relative strength of secondary velocity components to the streamwise velocity components (Banko et al., 2015). Regions with high values of $E$ would more rapidly produce lateral dispersion relative to the rate of streamwise transport.

These parameters calculated from the MRV measurements are plotted in Figures 10-15 for several characteristic locations. It should be noted that MRV does not resolve near wall gradients, and so $I_{1}$ and $D$ will tend to be underestimated. The effect on $I_{2}$ and $E$ is not as obvious. While it is expected that the no slip condition should limit the strength of near wall secondary flows, there are occasionally appreciable components in 

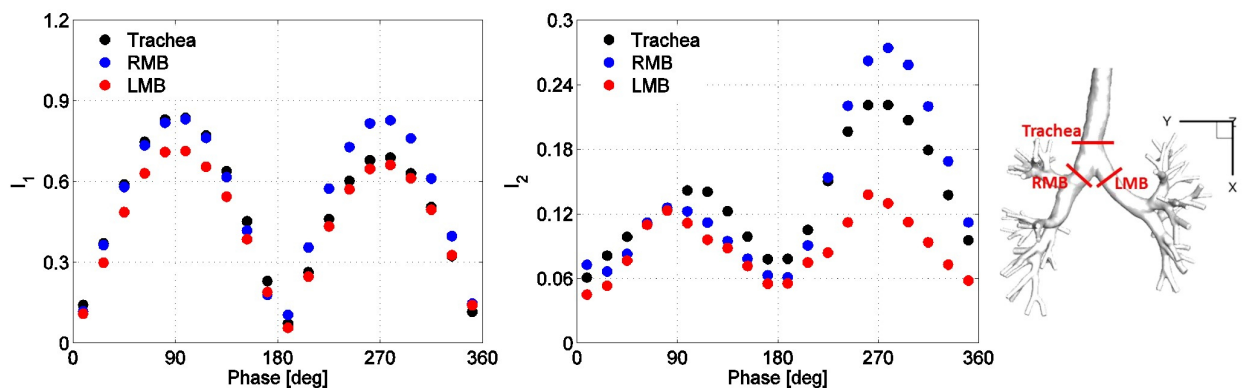

Figure 10: Integral parameters $I_{1}$ and $I_{2}$ at the first bifurcation, including the trachea, right main bronchus (RMB), and left main bronchus (LMB).
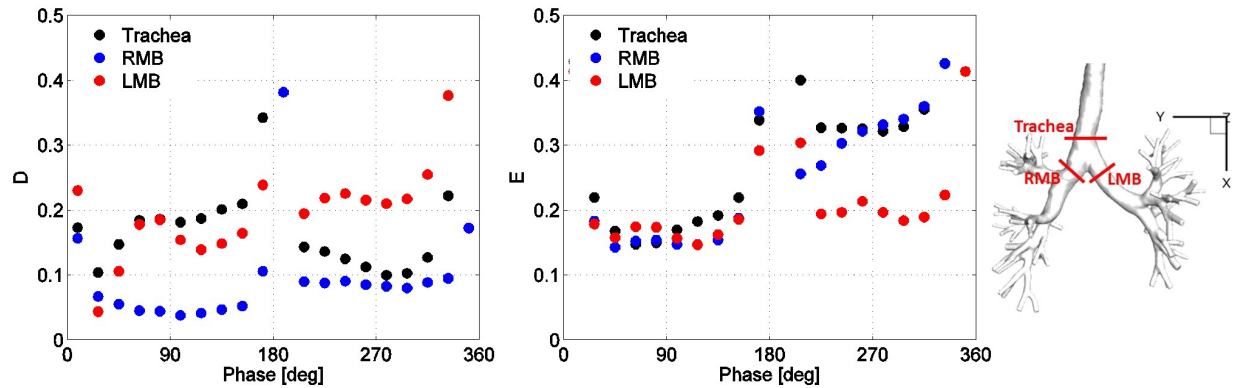

Figure 11: Integral parameters $D$ and $E$ at the first bifurcation, including the trachea, right main bronchus (RMB), and left main bronchus (LMB).
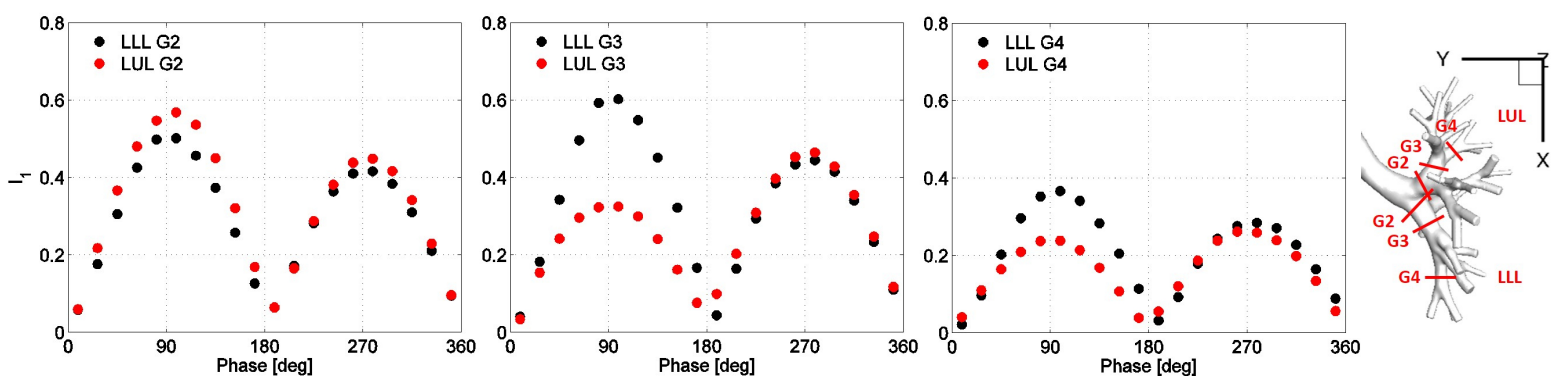

Figure 12: Integral parameter $I_{1}$ as a function of respiration phase along two bronchial paths. Nomenclature: left lower lobe (LLL), left upper lobe (LUL), generation number (G2, G3, G4).
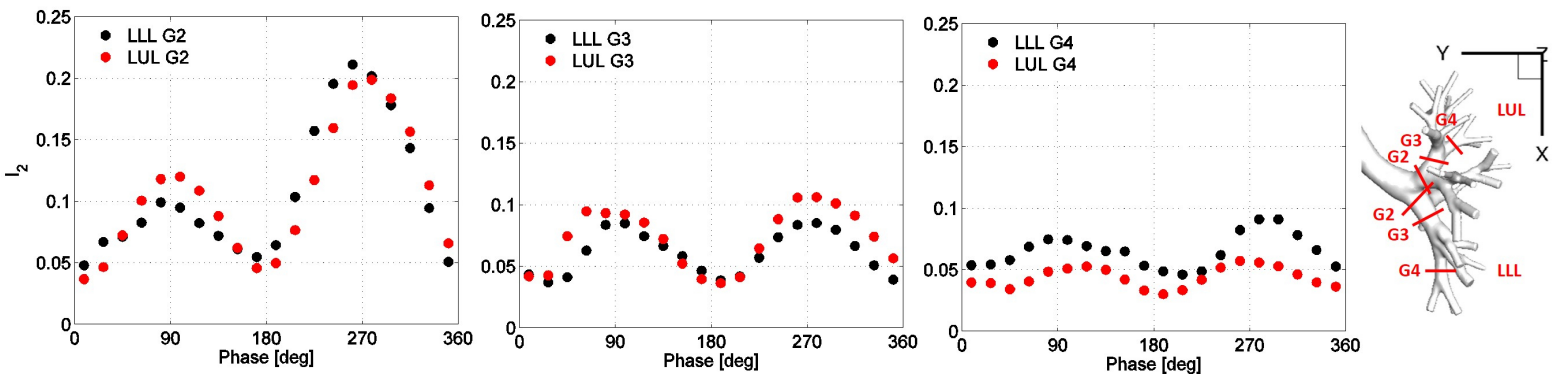

Figure 13: Integral parameter $I_{2}$ as a function of respiration phase along two bronchial paths. Nomenclature: left lower lobe (LLL), left upper lobe (LUL), generation number (G2, G3, G4). 

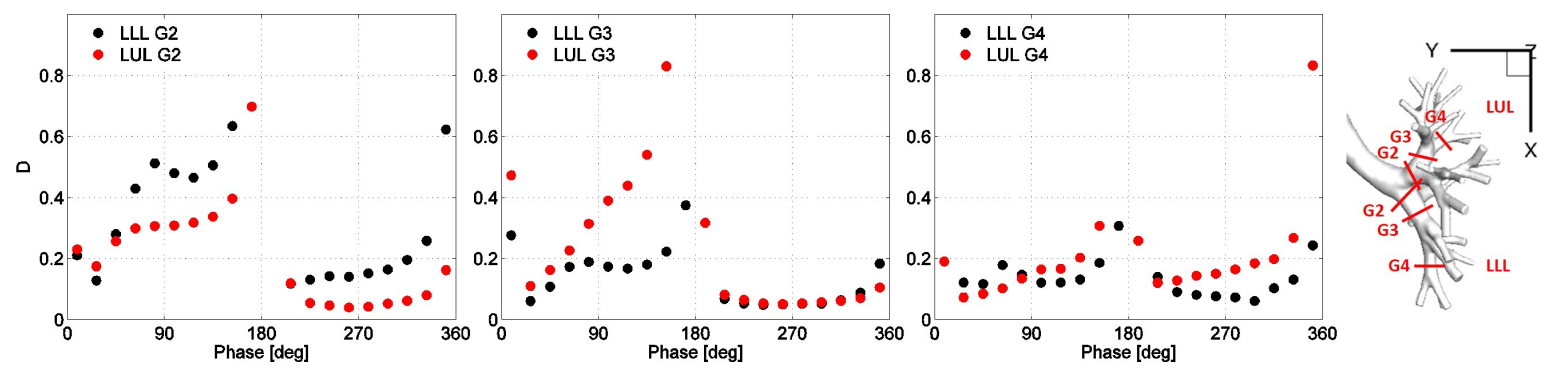

Figure 14: Integral parameter $D$ as a function of respiration phase along two bronchial paths. Nomenclature: left lower lobe (LLL), left upper lobe (LUL), generation number (G2, G3, G4).
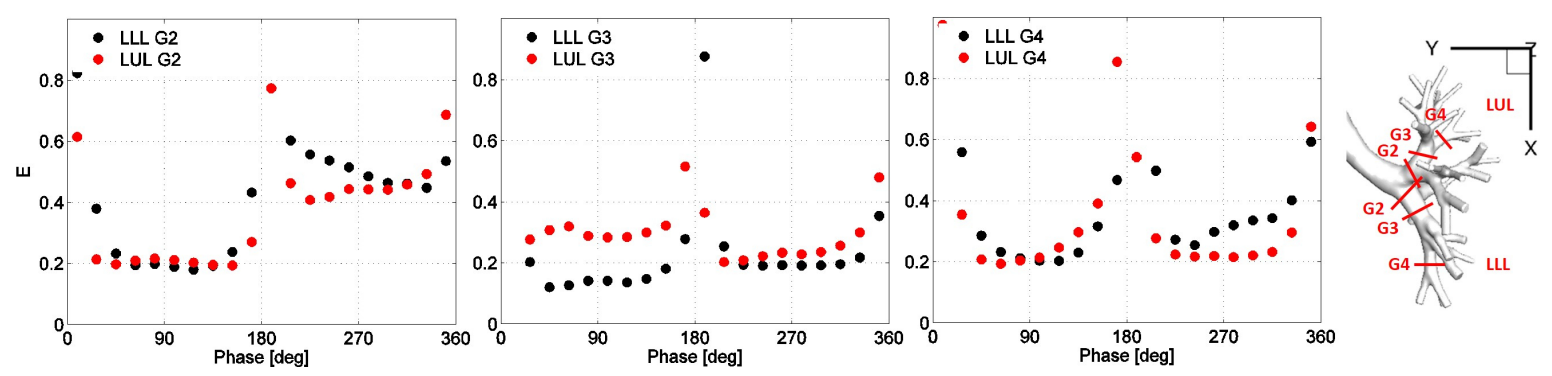

Figure 15: Integral parameter $E$ as a function of respiration phase along two bronchial paths. Nomenclature: left lower lobe (LLL), left upper lobe (LUL), generation number (G2, G3, G4).

near wall regions of reverse flow likely due to the influence of transverse pressure gradients. Also, due to the uncertainty in the measurements the approximate noise floor for the integral parameters is 0.07 . The parameters are not calculated in the terminal bronchial generations due to insufficient resolution and to avoid inflow effects from the plena during exhalation.

$I_{1}$ and $I_{2}$ increase and decrease approximately in phase with the flow rate throughout the breathing cycle. As a result, both streamwise and transverse dispersion by the bulk flow should be maximum near peak inhalation and exhalation as expected. The precise phase agreement varies across bronchial path and generation number suggesting that local flow features may lag or lead the variation in bulk flow rate. This also reflects the asymmetry of secondary flows about peak inhalation and exhalation, and is consistent with the regime diagram in Figure 3 since the transition from unsteady-convective zone IIIb to the viscous-convective zone IIIa occurs between generations two and three. The precise values of $D$ and $E$ depend on the local features of the anatomy such as branching angle, generation length, and expanding/contracting cross-sectional area in the streamwise direction. The overall trend is for $D$ to tend towards the laminar pipe flow value as generation number increases (recall that it will be underestimated), however local variations in time and between generations can be significant. The secondary flows can remain relatively strong with respect to the streamwise component throughout the first few bronchial generations, and $E$ does not necessarily monotonically decrease with increasing generation number. $D$ and $E$ show the most deviation from global trends in generations with realistic anatomical features that differ strongly from idealized ones such as large branching angle and short generation length. The parameters are sensitive to the flow oscillation in these regions.

Figure 10 plots the integral parameters $I_{1}$ and $I_{2}$ in the trachea, left main bronchus, and right main bronchus. $I_{1}$ is symmetric during inhalation and exhalation for the left and right main bronchi, but decreases by about $15 \%$ in the trachea during exhalation. The merging of the flows from the two main bronchi produces a m-shaped velocity profile which has a more uniform distribution across trachea (Zhang and Kleinstreuer, 2002). The secondary flow strength $\left(I_{2}\right)$ in the trachea during inhalation is higher for the decelerating phases than for the accelerating phases. This is in agreement with the vector fields examined in the previous section. The flow is in the unsteady convective regime (IIIb) and the vector fields show that the secondary flows leading to peak inhalation are not yet developed. After peak inhalation, the strong secondary flow structure lingers during the decelerating phases. $I_{2}$ is symmetric between inhalation and exhalation for the left main bronchus while it increases dramatically during exhalation in the right main bronchus and trachea. The secondary flows in the right main bronchus are strongly driven by the stream merging at high branching angle from the right upper lobe. Due to the short length of the right main bronchus, the circulation is carried into the lower trachea without significant decay, and dominates the weaker secondary components coming from the left main bronchus.

$D$ and $E$ are plotted for the same planes in the trachea and main bronchi in Figure 11. Overall, the trends are in agreement with the qualitative conclusions based on the vector fields. $D$ is higher in the trachea during inhalation than during exhalation because the momentum distribution during inhalation is skewed towards the anterior of the trachea in a crescent-like shape. The values of $D$ in the right main bronchus are relatively low due to 
the alignment of the trachea with this generation. The values of $E$ increase significantly in the right main bronchus and the trachea during exhalation due to the strong vortex generated by the flow merging from the left upper lobe at a high branching angle. It is interesting to note that, apart from the phases surrounding flow reversal where $D$ and $E$ may be undefined, the values of these integral parameters are relatively constant during inhalation and during exhalation. This suggests that the shape of the streamwise velocity profile is not drastically changing as the flow accelerates and decelerates, and that the secondary flows mostly increase and decrease in proportion to the streamwise components. Zhang and Kleinstreuer (2002) computed another integral measure of secondary flow strength in symmetric planar and $90^{\circ}$ non-planar bifurcation models with similar conclusions. A notable exception is the continual increase of $E$ in the right main bronchus during exhalation. The swirl generated by the jetting motion of the fluid from the right upper lobe does not readily decay as the streamwise flow decelerates.

Figures 12 and 13 plot $I_{1}$ and $I_{2}$, respectively, along two bronchial paths. Both decrease overall from the trachea to the fourth bronchial generation; however, the progression is not necessarily monotonic and depends on the details of the local anatomy. This is consistent with the conclusion from idealized models that the local bifurcation geometry and inlet condition from the parent generation dictate the flow structure in the daughter generation (Fresconi and Prasad, 2007; Comer et al., 2001; Zhang and Kleinstreuer, 2002). Similarly, $I_{1}$ and $I_{2}$ do not take the same values along independent bronchial paths as shown in Figure 12, nor are they symmetric between inhalation and exhalation. The lack of symmetry between inhalation and exhalation was not observed at lower Reynolds and Womersley numbers by Fresconi and Prasad (2007), but was observed at higher Reynolds and Womersley numbers in the study of Zhang and Kleinstreuer (2002). Such behavior is found where the local anatomy has a non-planar bifurcation, large branching angle, and/or quick expansion of the cross-section. It is also observed when the upstream flow influences the downstream generation. For example, $I_{1}$ at the third generation in the left lower lobe achieves a large value similar to that in the left main bronchus. Here the high momentum fluid along the inside wall of the left main bronchus passes through the relatively short second generation and is aligned with the third generation thereby producing a high momentum flux. Zhang and Kleinstreuer (2002) observed similar behavior in the planar Weibel model; however, due to the longer bronchial lengths the flow in the daughter generation was influenced only by the streamwise momentum distribution in the immediate upstream generation.

$D$ and $E$ are plotted for the same bronchial paths in Figures 14 and 15. Again, $D$ is relatively constant throughout much of the breathing cycle except in generations with anatomical features capable of producing strong inertial flow effects. Generations two through four of the bronchial tree transition from zone IIIb to IIIa of the regime diagram, and therefore a combination of unsteady and quasi-steady flow features is expected. As an example of unsteady flow, the cross-sectional area of generation two in the left lower lobe expands rapidly and is susceptible to flow separation during inspiration. A large region of reverse flow forms along the posterior wall of the bifurcation by peak inhalation (Banko et al., 2015). Due to the increasing adverse pressure gradient during flow deceleration, the flow remains separated. $D$ continually increases in this generation as the flow accelerates during inhalation and remains high during deceleration. This region of flow separation, like the vorticity shed from the first bifurcation, responds to the flow oscillation in a way that cannot be captured by quasi-steady flow assumptions. Features such as rapidly diffusing cross-sectional area (occurring for generations with short length) and high branching angle appear in realistic anatomies but are often neglected by the idealized models previously discussed. Rather, these simplified models emphasize flow structures generated due to bifurcation curvature and the Dean mechanism. Similar conclusions hold for $E$, which describes the relative rate of lateral to streamwise dispersion by the phase-averaged flow field. Depending non-trivially on the position in the bronchial tree and phase of the breathing cycle, this quantity can be as high as 0.5 during exhalation in generation two of the left lower lobe, or as low as 0.14 in the trachea and generation three of the left lower lobe during inspiration. Fresconi and Prasad (2007) did not observe secondary flow velocities higher than $20 \%$ of the streamwise component in the symmetrically branching airway model, although the study was conducted at lower Reynolds number. Zhang and Kleinstreuer (2002) observed larger secondary flow strength for some bronchial generations in the non-planar bifurcation model as compared to the planar bifurcation model, yet the maximum secondary flows remained below $20 \%$ of the streamwise during inhalation and exhalation. This suggests that features of real anatomies may produce more efficient lateral dispersion throughout the breathing cycle than idealized representations of the airways.

It is difficult to draw conclusions from the integral parameters $I_{1}$ and $I_{2}$ during the phases surrounding flow reversal due to the uncertainty in the velocity components. The calculated values are of the same order as the estimated root mean square noise. From examination of the vector fields, coherent secondary flow structures appear to persist through flow reversal while the streamwise component undergoes a zero crossing; however, measurements with reduced uncertainty and higher resolution in the distal bronchi are required to draw a definitive conclusion. Therefore comparisons cannot be made to the counter-flow phenomena studies of Choi et al. (2010), Grosse et al. (2007), and Adler and Brucker (2007).

\section{Conclusions}

The time-varying flow field through an anatomically accurate model of the human airways was studied at trachea based Reynolds and Womersley numbers relevant to breathing regimes during moderate exertion. Magnetic Resonance Velocimetry was used to obtain the three-dimensional, threecomponent, phase-averaged velocity field throughout the entire volume from the mouth through several generations of bronchial branching. In addition to qualitatively describing the flow features, integral parameters are defined to quantify the degree of velocity profile non-uniformity (which correlates with 
axial dispersion) and secondary flow strength (which correlates with lateral dispersion).

It is found that the extrathoracic airways significantly modify the tracheal flow and that the flow at the first bifurcation is highly asymmetric. This is in good agreement with previous studies on subject-specific anatomies, and is not captured by the first bifurcation in idealized models. Additionally, the integral measures of momentum distortion and secondary flow strength may behave non-monotonically between successive bronchial generations and can vary across independent bronchial paths. Broadly, this corroborates the conclusion from studies in idealized branching airways that the local bifurcation attributes (bifurcation curvature, and flow deflection by the carinal ridge) and inlet flow from the immediate upstream generation largely determine local flow structures. However, the details of the flow may be qualitatively and quantitatively different from what is found in idealized airway geometries. For example, the secondary flow structure in the left main bronchus during inhalation is dominated by the vorticity shed from the region of separation at the first bifurcation. Integral measures of secondary flow in the present anatomy demonstrate that the average secondary flow strength can easily exceed $20 \%$ of the streamwise flow strength up to generation four of the bronchi. Although the idealized model of Zhang and Kleinstreuer (2002) with non-planar bifurcations shows increased secondary flow strength in some generations compared to the planar bifurcation model, 20\% still appears to be an upper bound for the simplified anatomy. This suggests that realistic anatomies may produce enhanced lateral mixing by secondary flows throughout the breathing cycle.

The effect of flow oscillation is found to produce time dependent flow features which are asymmetric with respect to the acceleration and deceleration periods surrounding peak inhalation and exhalation. Qualitatively, the evolution of flow structures is in agreement with the regime diagram of Jan et al. (1989) which was developed in a model bifurcation. Secondary flows increase roughly in proportion with the local streamwise momentum, as observed in the integral parameters, with delayed onset of the secondary structure observed at peak inhalation/exhalation. However, there are notable exceptions: flow separation due to large streamline curvature at the first bifurcation, separation due to short generation length with rapidly diffusing cross-sectional area in the bronchial tree, and strong swirl generated in the right main bronchus as a result of flows merging at disparate angles during exhalation. The effect of flow oscillation is pronounced for these flow features, which are sensitive to branching angle, generation length, and other realistic features found in realistic anatomies as opposed to idealized models.

While the study of a single human anatomy is by definition not universal, a healthy adult male of average height and build is likely to posses more representative features than idealized models. It is imperative to understand the dependence of flow features on the real human anatomy in order to predict the flow, understand mixing, and improve medical devices such as mechanical ventilators.

\section{Acknowledgements}

This research was supported in part by the US Army Research Laboratory, through the Army High Performance Computing Research Center, Cooperative Agreement W911NF-070027. This material is also based upon work supported by the National Science Foundation Graduate Research Fellowship. Any opinion, findings, and conclusions or recommendations expressed in this material are those of the author(s) and do not necessarily reflect the views of the National Science Foundation.

\section{References}

Adler, K., Brucker, C., 2007. Dynamic flow in a realistic model of the upper human lung airways. Exp. Fluids 43, 411-423.

Banko, A., Coletti, F., Schiavazzi, D., Elkins, C., Eaton, J., 2015. Threedimensional inspiratory flow in the upper and central human airways. Exp. in Fluids 56, 117-128.

Beatty, P., Brau, A., Chang, S., 2007. A method for autocalibrating 2-d accelerated volumetric parallel imaging with clinically practical reconstruction times. In: Proceedings of the International Society for Magnetic Resonance. Vol. 15. Berlin, Germany, p. 1749.

Brau, A., Beatty, P., Skare, S., Bammer, R., 2008. Comparison of reconstruction accuracy and efficiency among autocalibrating data-driven parallel imaging methods. Magn. Reson. Med. 59, 382-395.

Briant, J., Cohen, B., 1989. Flow distribution through human and canine airways during inhalation and exhalation. J. Appl. Phys. 67, 1649-1654.

Brouns, M., Verbanck, S., Lacor, C., 2007. Influence of glottic aperture on the tracheal flow. J. Biomech. 40, 165-172.

Bulusu, K., Hussain, S., Plesniak, M., 2007. Determination of secondary flow morphologies by wavelet analysis in a curved artery model with physiological inflow. Exp. Fluids 55, 1-20.

Choi, J., Tawhai, M., Hoffman, E., Lin, C., 2009. On intra-and intersubject variabilities of airflow in the human lungs. Phys. Fluids 21, 101901.

Choi, J., Xia, G., Tawhai, M., Hoffmann, E., Lin, C., 2010. Numerical study of high-frequency oscillatory air flow and convective mixing in a ct-based human airway model. Annals of Biomedical Engineering 38, 3550-3571.

Comer, J., Kleinstreuer, C., Zhang, Z., 2001. Flow structures and particle deposition patterns in double-bifurcation airway models. part 1. air flow fields. J. Fluid Mech. 435, 25-54.

de Rochefort, L., Vial, L., Fodil, R., Maitre, X., Louis, B., et al., 2007. In vitro validation of computational fluid dynamic simulation in human proximal airways with hyperpolarized ${ }^{3}$ he magnetic resonance phase-contrast velocimetry. J. Appl. Phys. 102, 2012-2023.

Elkins, C., Alley, M., 2007. Magnetic resonance velocimetry: applications of magnetic resonance imaging in the measurement of fluid motion. Exp. Fluids 43, 823-858.

Fresconi, F., Prasad, A., 2007. Secondary velocity fields in the conducting airways of the human lung. J. Biomech. Eng. Trans. ASME 129, 722-732.

Grosse, S., Schroder, W., Klaas, M., Klockner, A., Roggenkamp, J., 2007. Time resolved analysis of steady and oscillating flow in the upper human airways. Exp. Fluids 42, 955-970.

Jan, D., Shapiro, A., Kamm, R., 1989. Some features of oscillatory flow in a model bifurcation. Journal of Applied Physiology 61, 147-159.

Kleinsteuer, C., Zhang, Z., 2010. Airflow and particle transport in the human respiratory system. Annu. Rev. Fluid Mech. 42, 301-334.

Lambert, A., O'shaughnessy, P., Tawhai, M., Hoffman, E., Lin, C., 2011. Regional deposition of particles in an image-based airway model: large-eddy simulation and left-right lung ventilation asymmetry. Aerosol Science and Technology.

Lieber, B., Zhao, Y., 1998. Oscillatory flow in a symmetric bifurcation airway model. Annals of Biomedical Engineering 26, 821-830.

Longest, P., Holbrook, L., 2012. In silico models of aerosol delivery to the respiratory tract - development and applications. Advanced Drug Delivery Reviews 64, 296-311.

Lyne, W., 1971. Unsteady viscous flow in a curved pipe. J. Fluid Mech. 45, 13-31. 
Markl, M., Chan, F., Alley, M., et al., 2003. Time-resolved three-dimensional phase-contrast mri. Journal of Magnetic Resonance Imaging 17, 499-506.

Padilla, A., 2012. The effect of upstream perturbations on $3 d$ annular diffusers. Ph.D. thesis, Stanford University.

Pelc, N., Sommer, F., Li, K., Brosnan, T., Herfkens, R., et al., 1994. Quantitative magnetic resonance flow imaging. Magn. Reson. Q 10, 125-147.

Shinneeb, A., Pollard, A., 2012. Investigation of the flow physics in the human pharynx/larynx region. Exp Fluids 53, 989-1003.

Soodt, T., Pott, D., Klaas, M., Schroeder, W., 2013. Analysis of basic flow regimes in a human airway model by stereo-scanning piv. Exp. Fluids 54, $1-10$.

Stapleton, K., Guentsch, E., Hoskinson, M., Finlay, W., 2000. On the suitability of $k-\epsilon$ modelling for aerosol deposition in the mouth and trhoat: a comparison with experiment. J. Aerosol Sci. 31, 739-749.

Wassermann, F., Hecker, D., Jung, B., Markl, M., Seifert, A., Grundmann, S., 2013. Phase-locked 3d3c-mrv measurements in a bi-stable fluidic oscillator. Exp. Fluids 54, 1487-1502.

Weibel, E., 1963. Morphometry of the human lung. Academic Press, New York. Yin, Y., Choi, J., Hoffman, E., Tawhai, M., Lin, C., 2013. A multiscale mdct image-based breathing lung model with time-varying regional ventilation. J. Comput. Phys. 244, 168-192.

Zhang, Z., Kleinstreuer, C., 2002. Transient airflow structures and particle transport in a sequentially branching lung airway model. Phys. Fluids 14, $862-880$.

Zhang, Z., Kleinstreuer, C., Hyun, S., 2012. Size-change and deposition of conventional and composite cigarette smoke particles during inhalation in a subject-specific airway model. J. Aerosol Sci. 46, 34-52. 